\section{Aspectos histomorfométricos do nervo laríngeo superior}

\author{
Romualdo Suzano Louzeiro Tiago ${ }^{1}$, \\ Mário Sérgio Lei Munhoz ${ }^{2}$, Flávio Paulo de Faria ${ }^{3}$, \\ Arnaldo Guilherme
}

Resumo

I

ntrodução: A laringe desempenha funções primordiais para a manutenção da vida do indivíduo. A perfeita integração entre as suas vias aferentes e eferentes, proporcionada pelo nervo laríngeo superior (NLS) e pelo nervo laríngeo recorrente, evita a aspiração de corpos estranhos e através de reflexos desencadeados pela movimentação da onda mucosa mantém o tônus da musculatura intrínseca da laringe. Distúrbios nessas vias neurais podem predispor à aspiração e pneumonias, bem como podem ser responsáveis por alterações na voz do indivíduo; condições estas mais freqüentes no idoso. Objetivo: Avaliar as características histomorfométricas do NLS em indivíduos idosos e comparar os achados com indivíduos adultos. Material e Método: Um fragmento desse nervo dos lados direito e esquerdo foi obtido a partir de cadáveres durante o exame de necropsia. Foram montados dois grupos de diferentes faixas etárias. Um grupo denominado Adulto composto por 5 indivíduos com média etária de 39,6 anos e um grupo Idoso composto por 6 indivíduos com média etária de 71,7 anos. A avaliação do material foi realizada em microscópio de luz com objetiva de $40 \mathrm{X}$ ligado a um sistema analisador de imagens computadorizado e foram obtidos os principais parâmetros relacionados ao nervo: freqüência das fibras mielínicas, densidade de fibras por $\mathrm{mm}^{2} \mathrm{e}$ a área intraperineural. Resultados: Foi observada uma redução estatisticamente significante das fibras mielínicas de menor diâmetro (1 e 2 mm) no grupo Idoso. Não houve diferença na freqüência de fibras mielínicas entre os lados direito e esquerdo, em ambos os grupos. Conclusão: Como funcionalmente as fibras mielínicas de menor diâmetro do nervo laríngeo superior são responsáveis pela sensibilidade da supraglote e pelo reflexo de fechamento glótico, a redução das fibras mielínicas no idoso pode estar relacionada, além de outros fatores, a uma maior predisposição à aspiração e às alterações de voz.

\section{Histomorphometryc aspects of the superior laryngeal nerve}

Palavras-chave: laringe, nervo laríngeo superior, disfagia, disfonia.

Key words: larynx, superior laryngeal nerve, dysphagia, voice disorders.

\section{Summary}

\begin{abstract}
Introduction: The larynx plays a primal role in the maintenance of life. The perfect integration between its afferent and efferent pathways, provided by the superior laryngeal nerve (SLN) and by recurrent laryngeal nerve, avoids foreign body aspiration and maintains the intrinsic laryngeal muscle tone by means of the reflexes elicited by the mucus wave movements. Aspirations and pneumonias as well as dysphonias, more frequently found among the elderly, might be triggered by disorders affecting these same neural pathways. Aim: The aim of this study was to assess the histomorphometryc characteristics of the SLN in elderly subjects in comparison to adults. Materials and method: A fragment of each side of the superior laryngeal nerve was obtained during necropsy examination and two different age groups were gathered. One group was made up of 5 adult subjects averaging 39,6 years of age and one was constituted of 6 elderly subjects averaging 71,7 years of age. The material was examined in a 40x objective lens microscope, connected to a computerized image analyzing system and the nerve's main parameters as the myelinated fibers frequency, fiber density per $\mathrm{mm}^{2}$, and intraperineural area were found. Results: A statistically significant reduction of the smaller myelinated fibers ( 1 and $2 \mathrm{~mm}$ ) was observed in the Elderly group. No difference was noted in the myelinated fiber frequency of the left/right sides. Conclusion: Being the low diameter superior laryngeal nerve myelinated fibers functionally responsible for the reflex of glotic closure, their reduction in the elderly subjects may be related, alongside with other factors, to a greater predisposition to aspiration and voice disorders.
\end{abstract}

\footnotetext{
Mestre em Otorrinolaringologia e Cirurgia de Cabeça e Pescoço pela Universidade Federal de São Paulo - Escola Paulista de Medicina 2 Professor Adjunto da Disciplina de Otoneurologia da Universidade Federal de São Paulo - Escola Paulista de Medicina.

3 Professor Adjunto do Centro de Microscopia Eletrônica da Universidade Federal de São Paulo - Escola Paulista de Medicina.

${ }^{4}$ Professor Adjunto da Disciplina de Otorrinolaringologia da Universidade Federal de São Paulo - Escola Paulista de Medicina.

Instituição: Departamento de Otorrinolaringologia e Distúrbios da Comunicação Humana e Centro de Microscopia Eletrônica (CEME) da Universidade Federal de São Paulo - Escola Paulista de Medicina.

Endereço para correspondência: Romualdo S. L. Tiago.Rua Estado de Israel, 493, apto 51 - Vila Clementino - São Paulo CEP 04022-001 - Tel. (0xx11) 5084.7725. - E-mail: romualdotiago@uol.com.br

Trabalho apresentado no II Congresso Triológico de Otorrinolaringologia, realizado em Goiânia-GO em agosto de 2001, tendo sido premiado como o melhor trabalho no módulo de Laringologia e Voz.

Artigo recebido em 30 de janeiro de 2002. Artigo aceito em 10 de fevereiro de 2002.
} 


\section{INTRODUÇÃO}

A laringe desempenha funções primordiais para a manutenção da vida do indivíduo. Suas principais funções em ordem de importância são: proteção das vias aéreas, respiração e fonação. A laringe protege as vias aéreas durante a deglutição, coordena e otimiza as vias aéreas superiores durante a respiração e promove o controle da fonação por meio de mecanismos intrínsecos em conjunto com a faringe e cavidades oral e nasal ${ }^{1,2}$.

Para a manutenção das funções da laringe é necessária uma perfeita integração entre as suas vias aferentes (sensibilidade) e eferentes (motora). O ramo interno do nervo laríngeo superior (NLS) é o responsável pela sensibilidade da região supraglótica até o nível da face superior das pregas vocais ${ }^{1,2,3} \mathrm{e}$ o ramo externo em associação com o nervo laríngeo recorrente (NLR) também é responsável pela sensibilidade da subglote ${ }^{4,5}$. As terminações nervosas mielínicas e amielínicas destes nervos formam um plexo na submucosa e são responsáveis pelos dois principais tipos de sensibilidade na laringe: mecanossensibilidade e quimiossensibilidade ${ }^{3}$. Impulsos aferentes dos receptores musculares profundos e das articulações cricotiróideas juntam-se às fibras que compõem o plexo submucoso para formar o ramo interno do NLS².

O NLS por meio de seu ramo externo é responsável pela inervação eferente do músculo cricotiróideo que tenciona e aproxima as pregas vocais e, através de um ramo comunicante entre o ramo externo e o NLR, também é responsável por parte da inervação motora do músculo tireoaritenóideo ${ }^{4,5}$. Os outros músculos intrínsecos da laringe são inervados por fibras mielínicas eferentes que trafegam pelo NLR. O músculo interaritenóideo parece ter uma inervação eferente mista a partir do ramo interno do NLS e do NLR ${ }^{4,5}$.

A proteção das vias aéreas através do reflexo de fechamento glótico é uma função básica e involuntária que depende de um bom funcionamento das vias aferentes e eferentes da laringe, evitando a aspiração durante o mecanismo da deglutição ${ }^{6}$. O indivíduo idoso tem maior predisposição à diminuição do reflexo de proteção das vias aéreas e conseqüentemente tem predisposição à aspiração de corpos estranhos e às pneumonias, 7 .

A contribuição respiratória da laringe foi melhor descrita por Negus (1949) que observou que a glote abrese em frações de segundos antes da passagem do ar desencadeado pela descida do diafragma. A função fonatória é a mais complexa e provavelmente a menos compreendida das três funções, embora seja a mais estudada. A fonação resulta da produção de um tom fundamental que é modificado pelas cavidades ressonantais que correspondem às vias aerodigestivas superiores. Um dos mecanismos de regulação da freqüência fundamental, além de regulação motora, está relacionado à informação sensorial da laringe (mucosa, receptores articulares e terminações aferentes musculares). Através da informação sensorial na supraglote e subglote, cantores treinados são capazes de posicionar a laringe a fim de atingir a freqüência fundamental desejada antes da emissão de um som audível' ${ }^{2,9}$. A sensibilidade originada na subglote e na supraglote é responsável pelo desencadeamento deste reflexo.

No idoso as alterações de voz são bastante freqüentes e, de acordo com Hirano, Kurita, Sakaguchi (1989) $)^{10} \mathrm{e}$ Sato \& Hirano (1997) ${ }^{11}$, a presbifonia pode estar relacionada, além de outros fatores, a alterações na vibração na mucosa da prega vocal e atrofia muscular.

Levando em consideração a importância do NLS e seus ramos, tem-se procurado identificá-los e preservá-los durante procedimentos cirúrgicos na região cervical. A lesão do ramo externo do NLS durante tiroidectomias leva à perda da tensão da prega vocal, secundária à denervação do músculo cricotiróideo, e a incapacidade de atingir freqüências mais altas ${ }^{12,13}$. Nos casos de laringectomia parcial supraglótica e supracricóidea existe o cuidado em preservar o ramo interno, mantendo desta forma a sensibilidade no nível das aritenóides e mucosa do seio piriforme, o que facilita a reabilitação dos pacientes e diminui o risco de aspiração e disfagia no pós-operatório ${ }^{14}$.

Considerando a importância do nervo laríngeo superior no que se refere às suas funções de proteção das vias aéreas e controle do mecanismo da fonação, propusemo-nos a realizar este trabalho, uma vez que na prática clínica há queixas no idoso de disfonia, engasgos e disfagia, nem sempre com alterações aos exames disponíveis.

O objetivo deste estudo é avaliar as características histomorfométricas do nervo laríngeo superior em indivíduos idosos e comparar os achados com indivíduos adultos com a finalidade de identificar parâmetros morfológicos que possam fornecer subsídios para justificar as alterações mais freqüentemente encontradas em idosos como diminuição da sensibilidade da laringe e alterações de voz.

\section{MATERIAL E MÉTODO}

O projeto de pesquisa deste estudo foi avaliado e aprovado pelo Comitê de Ética em Pesquisa do Hospital São Paulo - UNIFESP e pela Comissão de Ética para Análise de Projetos de Pesquisa (CAPPesq) do Hospital das Clínicas da Faculdade de Medicina da USP para que fossem possíveis a obtenção e coleta do material no Serviço de Verificação de Óbitos da Capital (SVOC) da Universidade de São Paulo.

Foram coletados fragmentos de $1 \mathrm{~cm}$ do NLS dos lados direito e esquerdo de 11 cadáveres, submetidos a exame necrológico no Serviço de Verificação de Óbitos da Capital da USP, durante os meses de novembro a março de 2001. Os fragmentos foram coletados entre sete horas e 30 minutos a 19 horas e 30 minutos após o óbito, e em 
seguida colocados em solução fixadora para posterior análise no Centro de Microscopia Eletrônica (CEME) da UNIFESP/EPM.

Foram selecionados cadáveres da raça branca, sexo masculino e sem antecedentes para doenças como diabetes, alcoolismo, neoplasia maligna e emagrecimento súbito ${ }^{15}$. A exclusão dos cadáveres, levando em consideração estes fatores previamente citados, foi possível pela obtenção da história pregressa dos cadáveres a partir das informações fornecidas pelos familiares e que constavam na ficha de óbito. Foram formados dois grupos: grupo Adulto - indivíduos com idade menor ou igual a 40 anos, composto por cinco cadáveres; grupo Idoso - indivíduos com idade superior a 60 anos, composto por seis cadáveres.

Relacionamos na Tabela 1 as iniciais do indivíduo, idade, peso, altura, índice de massa corpórea, tempo transcorrido do óbito até a coleta do fragmento e causa do óbito.

\section{Obtenção do material}

Para a obtenção do material foi realizada dissecção cervical a partir da incisão arciforme, habitualmente realizada no exame necrológico, que se estendeu de ombro a ombro passando abaixo da fúrcula esternal. A dissecção foi realizada em um plano abaixo do músculo platisma até o ângulo da mandíbula, bilateralmente.

Após a identificação da borda anterior do músculo esternocleidomastóideo a dissecção se estendeu profundamente em direção aos grandes vasos cervicais até a localização do feixe vásculo-nervoso laríngeo superior e dos ramos interno e externo do NLS; o fragmento do nervo foi obtido a uma distância de $3 \mathrm{~cm}$ da membrana tiro-hióidea7. Esta dissecção foi realizada dos lados direito e esquerdo de cada cadáver.

Os fragmentos foram imediatamente colocados em frascos individuais, identificados, com solução fixadora Karnovsky modificado ${ }^{16}$, contendo glutaraldeído $2,5 \%$, paraformaldeído $2 \%$ em tampão cacodilato de sódio $0,1 \mathrm{M}$, $\mathrm{pH} 7,4$, à temperatura ambiente, por um período mínimo de duas horas. Durante a sua obtenção, foram cortados transversalmente (perpendicular ao eixo de comprimento do nervo) para que fosse possível a obtenção dos seguintes dados histomorfométricos: área total de secção transversal do nervo (que incluiu o epineuro e o perineuro), área intraperineural (área representativa do número de fibras mielínicas), número de fibras mielínicas e a categorização das fibras de acordo com os seus diâmetros.

\section{Preparação do tecido para microscopia de luz}

O preparo e a avaliação morfométrica dos espécimes foram realizados no CEME-UNIFESP/EPM. Após a fixação inicial com solução fixadora de Karnovsky (1965) modificada, os fragmentos foram submetidos às seguintes etapas:

1. Quatro lavagens sucessivas em solução tampão cacodilato de sódio 0,1 M, pH 7,4;

2. Pós-fixação com tetróxido de ósmio $2 \%$ em solução tampão cacodilato de sódio $0,1 \mathrm{M}, \mathrm{pH}$ 7,4;

3. Imersão em solução aquosa de acetato de uranila $0,5 \%$ por 30 minutos, seguida por duas lavagens rápidas com água destilada.

4. Desidratação nas seguintes concentrações crescentes de etanol: 70\%, 90\% e 100\%. Em seguida o material foi imerso em óxido de propileno por duas vezes.

5. Infiltração em mistura de resina tipo Araldite $502^{\circledR}$ :Óxido de Propileno, em concentrações de 1:3, 1:2, 1:1. Após esta etapa o material foi colocado em resina pura. Em seguida os fragmentos do tecido foram transferidos para os moldes de inclusão e orientados horizontalmente para permitir o corte transversal e levados para polimerização da resina em estufa a $60^{\circ} \mathrm{C}$ por 72 horas.

Os blocos obtidos após esta preparação foram aparados e cortados em ultramicrótomo de marca Leica ${ }^{\circledR}$, modelo Reichert Ultracuts, com navalhas de vidro, para obtenção de cortes semifinos de $0,3 \mu \mathrm{m}$ de espessura. Em seguida os cortes foram colocados em lâminas de vidro e corados com azul de toluidina a $1 \%$.

Tabela 1. Iniciais do indivíduo, idade, peso e altura, índice de massa corporal (IMC), período entre o óbito e a coleta do fragmento $(\Delta t)$ e causa do óbito.

\begin{tabular}{|c|c|c|c|c|c|}
\hline Indivíduo & Idade & Altura/Peso & IMC $\left(\mathrm{Kg} / \mathrm{m}^{2}\right)$ & $\Delta \mathrm{t}$ & Causa do óbito \\
\hline JBV & 40 & $1,80 / 85$ & 26,23 & 19h30 & Dissecção aguda aorta \\
\hline LCA & 40 & $1,80 / 78$ & 24,07 & $11 \mathrm{~h} 30$ & Edema pulmonar \\
\hline JRF & 38 & $1,80 / 80$ & 24,69 & $14 \mathrm{~h}$ & Rotura de aneurisma de aorta \\
\hline PCP & 40 & $1,90 / 88$ & 24,38 & $15 h$ & Infarto agudo do miocárdio \\
\hline CAM & 40 & $1,90 / 90$ & 24,93 & $12 \mathrm{~h}$ & Infarto agudo do miocárdio \\
\hline APJ & 79 & $1,80 / 86$ & 26,54 & $8 \mathrm{~h} 30$ & Infarto agudo do miocárdio \\
\hline MOF & 69 & $1,75 / 70$ & 22,87 & $7 \mathrm{~h} 30$ & Infarto agudo do miocárdio \\
\hline CRN & 68 & $1,80 / 70$ & 21,60 & $11 \mathrm{~h}$ & Infarto agudo do miocárdio \\
\hline DG & 65 & $1,80 / 75$ & 23,14 & $13 \mathrm{~h}$ & Crise asmática \\
\hline OAC & 65 & $1,80 / 70$ & 21,60 & $16 \mathrm{~h}$ & Infarto agudo do miocárdio \\
\hline $\mathrm{BC}$ & 81 & $1,75 / 67$ & 21,89 & $11 \mathrm{~h}$ & Infarto agudo do miocárdio \\
\hline
\end{tabular}




\section{Estudo morfométrico}

Os cortes foram avaliados em microscópio de luz da marca Nikon ${ }^{\circledR}$, modelo Optiphot II, com objetiva de 10x e 40x e aumento final de 215 e 860 vezes, acoplado a um sistema analisador de imagens, de marca Leica ${ }^{\circledR}$, modelo Quantimet 500 QWIN. Nesse sistema as imagens do campo avaliado foram digitalizadas através de uma câmara de vídeo de alta resolução de marca Sony ${ }^{\circledR}$, modelo XC 003P e transferidas para o computador.

A avaliação morfométrica, através do microscópio de luz, foi dividida em duas etapas, descritas a seguir:

- Foi obtida a medida da área total da secção transversal e da área intraperineural do NLS. Para obter as medidas da área, as imagens do nervo foram digitalizadas a partir da objetiva de 10x, o que representou um aumento final de 215 vezes no monitor do computador. Através de um programa do computador foi possível obter o valor das áreas total e intraperineural. A obtenção da área intraperineural foi importante para o cálculo da densidade das fibras mielínicas por $\mathrm{mm}^{2}$ e do número total de fibras do nervo a partir de uma amostra representativa.

- Foram obtidos o número e o diâmetro externo das fibras mielínicas do NLS. O aumento final da imagem na tela do monitor para a objetiva de 40x foi de 860 vezes. Para obter a medida do diâmetro externo das fibras mielínicas essa imagem foi ampliada digitalmente duas vezes, o que forneceu um aumento final de 1.720 vezes. Foram avaliados cerca de dois a três campos aleatórios por lâmina. Desta forma foi avaliada uma área que variou de 6,13\% a 15,3\%, dependendo da lâmina. Conhecendo a área intraperineural obtida na primeira etapa e a área total dos campos avaliados, foram estimados o número total e a densidade numérica por $\mathrm{mm}^{2}$ das fibras mielínicas do NLS.

Para evitar erro de amostragem (efeito de margem) foram excluídas as fibras mielínicas projetadas sobre as linhas inferior e esquerda que delimitam o $\mathrm{campo}^{17}$. O menor diâmetro da fibra (a maior distância perpendicular ao maior eixo da fibra mielínica) foi escolhido para a medida das fibras mielínicas que tinham um perímetro elíptico ou irregular ${ }^{7,18}$.

\section{Análise estatística}

As distribuições das variáveis foram representadas pela freqüência, média e desvio padrão. Para a comparação entre as idades e o índice de massa corpórea (IMC) foi usado o teste t de Student. Para a comparação das médias da área intraperineural, densidade e freqüência das fibras mielínicas entre os grupos Adulto e Idoso foi usado o método estatístico Análise de Variância com Medida Repetida. Adotamos o nível de significância $\alpha$ de 0,05 . O valor de p (probabilidade de ocorrer um evento) $<0,05$ foi considerado como significante, levando à rejeição da hipótese de igualdade entre os grupos.

As análises estatísticas foram realizadas na Disciplina de Bioestatística do Departamento de Medicina Preventiva da UNIFESP-EPM, utilizando o programa estatístico SAS v. 6,12 (SAS/STAT Software, 1997: Release 6,12 Cary, NC: SAS Institute Inc.).

\section{RESULTADOS}

Na Tabela 2 observamos, através do teste t de Student com variâncias desiguais, que existe diferença estatisticamente significante entre as médias de idade dos dois grupos ( $\mathrm{p}<0,001)$. O grupo Idoso é em média 31,57 $\pm 2,91$ anos mais velho do que o grupo Adulto.

Na Tabela 3 observamos, através do teste t de Student, que a média do índice de massa corporal do grupo Adulto é igual a do grupo Idoso $(\mathrm{p}=0,065)$.

De acordo com o Gráfico 1, na comparação das áreas intraperineurais do grupo Adulto e Idoso utilizando o modelo estatístico de Análise de Variância com Medidas Repetidas, não existe diferença estatisticamente significante entre os grupos ( $p=0,9840)$; no entanto existe diferença estatisticamente significante entre a área média do lado direito e a área média do lado esquerdo $(\mathrm{p}=0,0080)$. A área do lado esquerdo é em média $51215,9 \pm 14682,5 \mu \mathrm{m}^{2}$, ou 0,0512 $\pm 0,0147 \mathrm{~mm}^{2}$, maior do que a área do lado direito.

De acordo com o Gráfico 2, a comparação das densidades numéricas do grupo Adulto e Idoso, utilizando o modelo estatístico de Análise de Variância com Medidas Repetidas, não evidenciou diferença estatisticamente significante entre os grupos ( $\mathrm{p}=0,2042)$, entretanto, existe diferença estatisticamente significante entre a densidade numérica média do lado direito e a densidade numérica média do lado esquerdo ( $\mathrm{p}=0,0439)$. O lado direito tem em média 2158,2 \pm 369 ,9 fibras mielínicas $/ \mathrm{mm}^{2}$ a mais do que o lado esquerdo.

Tabela 2. Medidas descritivas da idade (anos) segundo os grupos.

\begin{tabular}{lcc}
\hline Idade (anos) & Adulto & Idoso \\
\hline Média & 39,60 & 71,17 \\
Desvio padrão & 0,89 & 7,05 \\
Mínima & 38 & 65 \\
Máxima & 40 & 81 \\
\hline
\end{tabular}

Teste $t$ de Student com variâncias desiguais $p<0,001$

Tabela 3. Medidas descritivas do índice de massa corpórea (IMC) segundo os grupos.

\begin{tabular}{lcc}
\hline IMC $\left(\mathrm{Kg} / \mathrm{m}^{2}\right)$ & Adulto & Idoso \\
\hline Média & 24,86 & 22,94 \\
Desvio padrão & 0,83 & 1,88 \\
Mínimo & 24,07 & 21,60 \\
Máximo & 26,23 & 26,54 \\
\hline
\end{tabular}

Teste $t$ de Student 
No Gráfico 3 observamos uma curva unimodal em ambos os grupos com uma maior freqüência de fibras mielínicas de menor diâmetro, sendo do lado direito mais freqüentes as fibras mielínicas de $1 \mu \mathrm{m}$ e do lado esquerdo as fibras mielínicas de $2 \mu \mathrm{m}$, sem diferença estatisticamente significante intragrupo $(\mathrm{p}=0,4298)$. Existe uma diferença estatisticamente significante na distribuição das freqüências das fibras mielínicas de acordo com os seus diversos diâmetros ( $\mathrm{p}=0,0001)$ em ambos os grupos, predominando as fibras com menores diâmetros. Não existe diferença estatisticamente significante entre os lados nos dois grupos quando se compara a distribuição das freqüências das fibras mielínicas nos seus diversos diâmetros ( $\mathrm{p}=0,4725$ ). Comparando os grupos isoladamente, não observamos diferença estatisticamente significante mas com uma tendência a existir esta diferença $(\mathrm{p}=0,0627)$.

As fibras mielínicas de menor diâmetro $(1 \mu \mathrm{m}$ e 2 $\mu \mathrm{m})$ são mais freqüentes no grupo Adulto $(\mathrm{p}=0,0001)$, sendo observado em média $429 \pm 86$ fibras mielínicas a mais que no grupo Idoso (Gráfico 4). Entre $1 \mu \mathrm{m}$ e $2 \mu \mathrm{m}$ existe uma semelhança no comportamento da distribuição das freqüências das fibras mielínicas ou paralelismo

Gráfico 1. Perfis das médias das áreas intraperineurais do NLS dos grupos Adulto e Idoso, segundo os lados direito e esquerdo

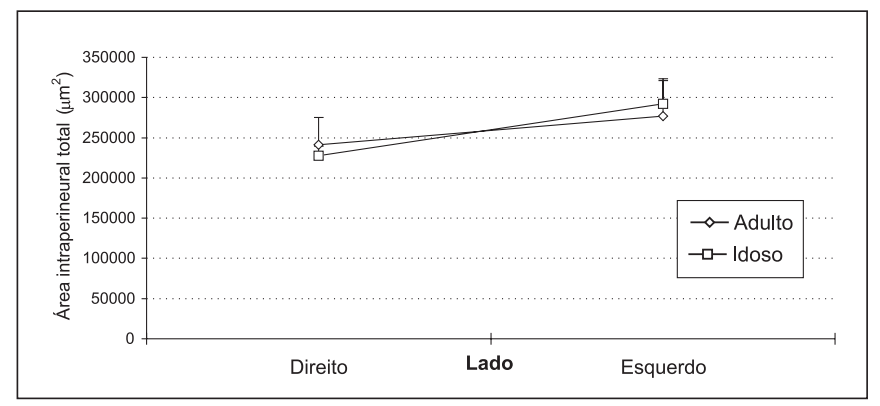

Teste Análise de Variância com Medidas Repetidas

Grupo* Lado $p=0,3446$; Grupo $p=0,9840$; Lado $p=0,0080$

Gráfico 3. Perfis das médias das freqüências das fibras mielínicas do NLS nos grupos Adulto e Idoso, lados direito e esquerdo

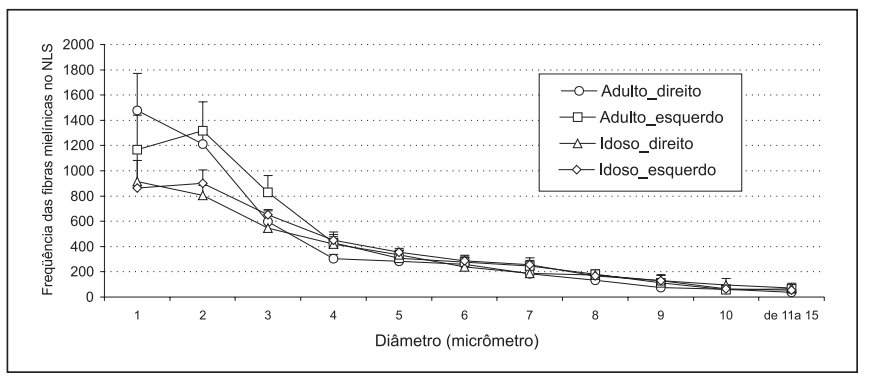

Teste Análise de Variância com Medidas Repetidas

Diâmetro* Lado $p=0,4725$; Diâmetro* Grupo $p=0,0326$

Lado intragrupo $p=0,4298$; Grupo $p=0,0627$; Diâmetro $p=0,0001$ $(\mathrm{p}=0,8508)$. Entre $2 \mu \mathrm{m}$ e $3 \mu \mathrm{m}$ deixa de existir o paralelismo no comportamento da distribuição das freqüências das fibras mielínicas $(p=0,0294)$ e a partir de $3 \mu \mathrm{m}$ a distribuição das freqüências passa a ser semelhante nos dois grupos $(\mathrm{p}=0,7730)$.

\section{DISCUSSÃO}

O processo de envelhecimento leva a alterações no organismo responsáveis pelos mais diversos tipos de manifestações a depender do órgão que está sendo avaliado. Observamos com certa freqüência que o idoso é mais predisposto a apresentar distúrbios de voz e de deglutição, sendo estas alterações associadas a certos graus de aspiração e conseqüentes pneumonias.

Com relação à vOz no idoso, diversos trabalhos têm abordado este assunto, principalmente no que diz respeito às alterações na movimentação das pregas vocais e na estrutura de algumas áreas da laringe $\mathrm{e}^{10,11}$. No entanto existem poucos trabalhos que avaliam a inervação da laringe sob o aspecto microscópico, correlacionando os achados com as diferentes faixas etárias.

Gráfico 2. Perfis das médias das densidades numéricas do NLS do grupo Adulto e Idoso, lado direito e esquerdo.

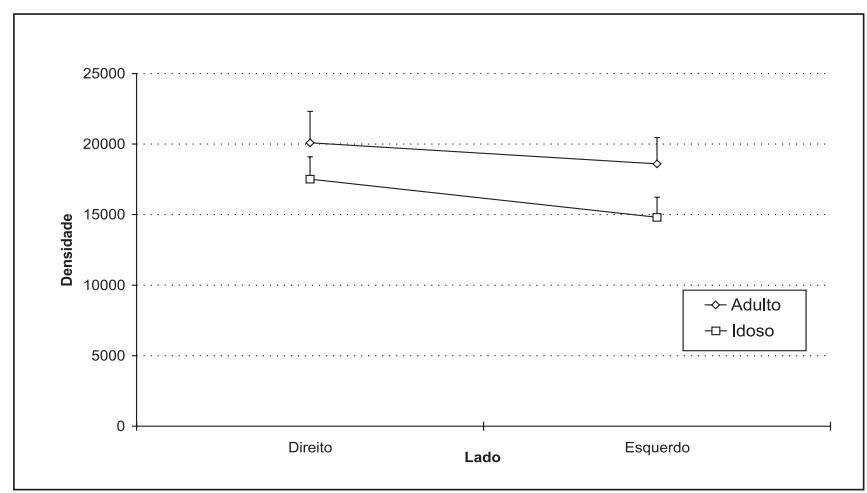

Gráfico 4. Paralelismo de distribuição entre as fibras mielínicas do NLS nos Grupos Adulto e Idoso

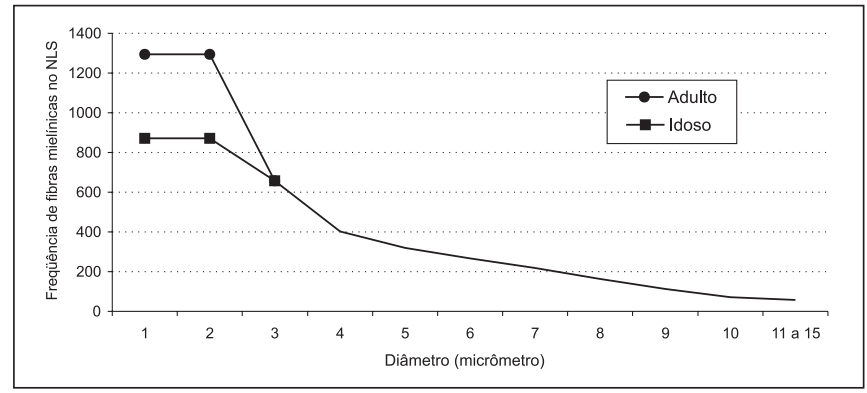

Paralelismo entre as fibras de 1 e $2 \mu \mathrm{m} \mathrm{p}=0,8508$

Paralelismo entre as fibras de 2 a $3 \mu \mathrm{m} \mathrm{p}=0,0294$ 
Os poucos trabalhos sobre a avaliação histomorfométrica do NLS e do NLR não seguem uma padronização sobre o método para obtenção do material e nem sobre o método de avaliação propriamente dito (contagem de fibras, mensuração dos diâmetros e área).

A investigação da freqüência das fibras mielínicas em indivíduos normais e em animais já foi realizada por alguns autores, apresentando resultados variados. Isto pode estar relacionado ao estudo de espécies diferentes e à falta de padronização do ponto de secção do nervo, informações nem sempre presentes nos trabalhos publicados ${ }^{19}$.

Os trabalhos publicados até o momento sobre a histomorfometria do NLS em humanos são compostos por um número reduzido de indivíduos e sem padronização sobre o modo de coleta e preparação do material o que dificulta a comparação dos resultados ${ }^{7,20,21}$.

Com o intuito de avaliar o NLS segundo suas características histomorfométricas, constituímos uma amostra de indivíduos que não apresentavam doenças pregressas que pudessem vir a interferir com o padrão normal para a idade. Desta forma na Tabela 1, que demonstra os grupos de indivíduos que constituíram nossa amostra, observamos que a principal causa do óbito em ambos os grupos foi o infarto agudo do miocárdio, seguido de rotura de aneurisma (mais freqüente em indivíduos mais jovens), edema pulmonar cardiogênico e crise asmática; todos considerados como causa de morte natural. Na composição da nossa amostra procuramos formar dois grupos com faixas etárias bem distintas.

Inicialmente tínhamos a intenção de formar um grupo adulto jovem com idade variando entre 20-30 anos o que evidenciaria mais facilmente possíveis diferenças com o grupo idoso acima de 60 anos de idade. Como a principal causa de óbito na faixa etária dos 20-30 anos é por morte violenta não nos foi possível o acesso a este grupo visto que esses cadáveres são encaminhados para o Instituto Médico Legal de São Paulo e a nossa coleta só foi autorizada no Serviço de Verificação de Óbitos da Capital (SVOC-USP), onde a necropsia é realizada em indivíduos com morte natural. Este fato impossibilitou o estudo em um grupo mais jovem, sendo as médias etárias dos nossos grupos de 39,60 anos para Adultos e 71,17 anos para Idosos, diferente portanto dos grupos de Mortelliti et al (1990) que trabalharam com médias etárias de 23,8 anos para adultos jovens e 76,2 anos para idosos. A causa de óbito no grupo Jovem destes autores foi principalmente o politraumatismo.

Foram excluídos desta nossa amostra os indivíduos portadores de doenças associadas ao desenvolvimento de polineuropatia periférica, como diabetes, alcoolismo, neoplasias, emagrecimento súbito e uso de quimioterápicos, como sugerem os trabalhos de Dyck (1989).

Não encontramos na literatura nenhum trabalho que tenha avaliado o NLS dos dois lados em indivíduos de diferentes faixas etárias. Mortelliti et al (1990) avaliaram-no apenas do lado direito. Levando em consideração que a principal função do NLS é a sensibilidade da supraglote e de parte da subglote $e^{1,2,3,4,5}$ e que não existe diferença de sensibilidade entre os dois lados da supraglote ${ }^{8,22}$, gostaríamos de verificar como se apresentam as fibras mielínicas no NLS bilateralmente e detectar se o padrão de distribuição das fibras mielínicas é ou não semelhante.

Na Tabela 2 observamos uma diferença estatisticamente significante $(\mathrm{p}<0,001)$ com relação às médias de idade entre os grupos Adulto e Idoso. O grupo Idoso é em média $31,57 \pm 2,9$ anos mais velho do que o grupo Adulto. Trabalhamos portanto com uma diferença de idade entre os grupos menor do que Mortelliti et al (1990) cuja diferença de idade entre os grupos foi de 52,4 anos.

Analisando a Tabela 3 observamos que não existe diferença estatisticamente significante no índice de massa corpórea entre os dois grupos. Mortelliti et al (1990) compararam a altura e o peso e não encontraram diferença estatisticamente significante entre os dois grupos Jovem (23,8 anos) e Idoso (76,2 anos).

Conforme informamos anteriormente, a nossa amostra foi composta de 11 cadáveres onde avaliamos ambos os lados, o que representou um total de 22 fragmentos. Cada fragmento constituiu-se em uma lâmina e em seguida foram feitas as análises no microscópio de luz ligado a um sistema analisador de imagens LEICA QWIN $^{\circledR}$. A análise das características morfométricas das fibras mielínicas através de um sistema computadorizado facilita a contagem e fornece resultados confiáveis e precisos, principalmente quando se trabalha com grandes amostras e existe a necessidade de serem avaliados muitos campos $^{19}$.

Muitos trabalhos têm estimado o diâmetro das fibras por meio da mensuração de todas as fibras mielínicas na secção transversal do nervo. Isto torna o trabalho muito difícil quando se pretende trabalhar com uma amostra maior, onde o número de fibras mielínicas por secção é grande, como no NLS que varia de 3.000 a 5.000 fibras.

A contagem das fibras mielínicas foi realizada a partir da amostragem aleatória de campos, sendo avaliado um mínimo de 6\% da área de secção de cada nervo, e em seguida foi estimada a freqüência total das fibras mielínicas para toda a área intraperineural. Mayhew \& Sharma (1984) mostraram as vantagens e limitações de se realizar estas medidas através de um sistema de contagem semiautomática. Pela avaliação estatística concluíram que em nervos com um número grande de fibras a coleta através de amostragem de $6 \%$ da área total é tão precisa quanto a medida de todas as fibras mielínicas. Além da eficiência na avaliação da freqüência das fibras, a amostragem de campos também está associada a um menor custo e a uma redução do tempo na realização do trabalho, diminuindo as chances de erro. 
Não encontramos diferença estatisticamente significante nas áreas intraperineurais entre os grupos Adulto e Idoso, no entanto observamos uma área intraperineural maior do lado esquerdo em ambos os grupos, estatisticamente significante $(\mathrm{p}=0,008)$, como mostra o Gráfico 1 . Portanto o NLS apresenta-se com área intraperineural igual no Adulto e no Idoso, e maior no lado esquerdo do que no lado direito. Mortelliti et al (1990) também não encontraram diferença entre as áreas, quando compararam as diferentes faixas etárias. Jacobs \& Love (1985) avaliaram o nervo sural em humanos e observaram um aumento na área de secção dependente da idade, decorrente do aumento de tecido conjuntivo e um maior espaçamento entre as fibras mielínicas observadas no idoso.

Com relação à densidade numérica das fibras mielínicas também não houve diferença estatisticamente significante entre os grupos. Observamos que a densidade do lado direito é maior do que a do lado esquerdo, com diferença estatisticamente significante $(\mathrm{p}=0,043)$, como mostra o Gráfico 2. Portanto o NLS apresenta-se com densidade semelhante no Adulto e no Idoso, e maior no lado direito do que no lado esquerdo. Na literatura observamos que Mortelliti et al (1990) também não encontraram diferença na densidade entre as diferentes faixas etárias.

No grupo Adulto a freqüência de fibras mielínicas do lado direito varia de 3.552 a 5.499 com média $4.631 \pm$ 825 , e no lado esquerdo varia de 2.546 a 5.960 com média $4.985 \pm 1.394$. No grupo Idoso a freqüência de fibras mielínicas do lado direito varia de 3.302 a 4.985 com média $3.920 \pm 623$, e no lado esquerdo varia de 3.304 a 5.386 com média $4.188 \pm 734$.

No Gráfico 3 podemos constatar um predomínio das fibras mielínicas de menor diâmetro em ambos os grupos. Temos então que no NLS há maior freqüência de fibras mielínicas de menor diâmetro (1 e $2 \mu \mathrm{m})$ tanto no Adulto quanto no Idoso, havendo nestes grupos uma simetria de distribuição entre os lados direito e esquerdo. Após a análise estatística dos resultados deste estudo verificamos que ocorre uma menor freqüência de fibras mielínicas de menor diâmetro (1 e $2 \mu \mathrm{m}$ ) no idoso, quando comparamos com indivíduos mais jovens. De acordo com a literatura, o NLS é composto predominantemente por fibras mielínicas de menor calibre (1 a $2 \mu \mathrm{m})^{7,20,21,25}$

Mortelliti et al (1990), na avaliação do NLS direito, também observaram uma diferença estatisticamente significante entre a freqüência de fibras mielínicas de menor calibre nos seus dois grupos. Dahlqvist (1982) não observou diferença na freqüência de fibras mielínicas entre os lados direito e esquerdo na avaliação do NLR em ratos. De acordo com os nossos achados este padrão também ocorre no NLS em humanos.

O diâmetro das fibras nervosas oferece uma base conveniente para a comparação de fibras entre diferentes grupos a serem pesquisados. O diâmetro externo das fibras tem sido usado para classificar as fibras mielínicas em diferentes grupos e correlaciona-se bem com as suas propriedades fisiológicas, como por exemplo, a velocidade de condução do estímulo nervoso ${ }^{23}$.

As fibras nervosas periféricas têm calibre variável, indo desde as fibras com grande diâmetro e intensamente mielínicas até as fibras muito delgadas e sem mielina. A condução do potencial de ação é mais rápida para as fibras mielínicas de maior calibre. A transmissão de estímulo nervoso para o músculo esquelético é realizada por fibras mielínicas de grande diâmetro, que variam de 8-16 $\mu \mathrm{m}$. A transmissão de estímulo sensorial pode ser realizada por fibras mielínicas e amielínicas ${ }^{27}$.

Segundo Burt (1995), os fisiologistas classificam as fibras nervosas sensoriais em quatro tipos:

- Tipo I: fibras mielínicas de grande diâmetro (12-20 $\mu \mathrm{m})$, que conduzem estímulos aferentes das terminações ânulo-espirais do fuso muscular e dos órgãos tendinosos de Golgi.

- Tipo II: fibras mielínicas de médio diâmetro (5-12 $\mu \mathrm{m})$, que conduzem estímulos aferentes das terminações "em buquê" dos fusos musculares e de receptores táteis cutâneos.

- Tipo III: fibras mielínicas de pequeno diâmetro (1-5 $\mu \mathrm{m})$, que conduzem estímulos aferentes relacionados ao tato grosseiro, temperatura e alguma sensação de dor aguda.

- Tipo IV: fibras amielínicas (0,5-2 $\mu \mathrm{m})$, que conduzem estímulos aferentes relacionados a tato grosseiro, temperatura e alguma sensação de dor crônica.

O nervo laríngeo recorrente (NLR) é o principal responsável pela inervação motora dos músculos intrínsecos da laringe e tanto na espécie humana quanto em outros animais é formado predominantemente por fibras mielínicas de maior diâmetro ${ }^{19,21,26,28}$. Harrison (1981) tendo avaliado o NLR do lado esquerdo observou que existe uma predominância das fibras mielínicas de maior diâmetro, quando comparada com o lado direito, e isso pode estar relacionado a uma velocidade de condução mais rápida do impulso nervoso já que este nervo possui um trajeto mais longo. Dahlqvist (1982) e Lima (1995) não encontraram diferença na freqüência das fibras mielínicas no NLR quando compararam os dois lados.

O uso de tecidos humanos permite a detecção de alterações inerentes ao processo de envelhecimento que não conseguimos detectar quando utilizamos modelos animais com período de sobrevida relativamente curto. A avaliação histomorfométrica de nervos sensoriais em outras espécies (ratos) não evidenciou diferenças estatisticamente significantes, quando foram comparadas diferentes faixas etárias ${ }^{18,25}$. Este mesmo estudo na espécie humana, que apresenta uma sobrevida maior, já detecta algumas diferenças. 
Jacobs \& Love (1985), em um estudo quantitativo do nervo sural, observaram diminuição da densidade das fibras mielínicas com o decorrer da idade, associada ao aumento no diâmetro das fibras, com maior separação entre estas fibras e o aumento da área de secção transversa no idoso e acreditam que a degeneração das fibras mielínicas é um evento comum após os 60 anos de idade.

Levando em consideração que funcionalmente as fibras mielínicas de menor calibre do NLS são sensoriais, responsáveis pela sensibilidade nas regiões da supraglote e subglote e associadas ao reflexo de fechamento súbito das pregas vocais e movimentos de deglutição, a redução do número deste tipo de fibras pode estar associada à diminuição da sensibilidade nesta região, com conseqüente predisposição para aspiração de corpos estranhos e para pneumonias de repetição nos idosos.

Os nossos achados, comparados com as observações da fisiologia dos nervos periféricos mencionadas, permitemnos inferir que o idoso por apresentar uma menor quantidade de fibras mielínicas, principalmente as de pequeno calibre, possui diminuição da sensibilidade na região inervada pelo NLS. A diminuição da sensibilidade da laringe pode também estar contribuindo para a diminuição do tônus reflexo dos músculos intrínsecos e o aparecimento das alterações vocais no idoso.

Estes achados devem ser confrontados com as alterações clínicas observadas, tais como: engasgos, diminuição da velocidade de deglutição, penetrações ou microaspirações que são mais freqüentes nesta faixa etária.

\section{CONCLUSÕES}

O estudo histomorfométrico do NLS em cadáveres de idosos quando comparado ao de adultos mostrou que o número total de fibras mielínicas é semelhante em ambos os lados nos dois grupos, sendo a área intraperineural do lado esquerdo maior que a área do lado direito, compensado por uma densidade de fibras mielínicas maior no lado direito que no lado esquerdo.

Observa-se uma diminuição estatisticamente significante de fibras mielínicas de menor diâmetro (1 e $2 \mu \mathrm{m}$ ) no NLS do idoso quando comparado com indivíduos mais jovens.

Os nossos achados somando à luz das observações dos autores nos permitem inferir que a diminuição da sensibilidade da laringe pode estar contribuindo para a diminuição do tônus reflexo dos músculos intrínsecos da laringe, aparecimento das alterações vocais e predisposição à disfagia e aspiração no idoso.

Estudos da correlação da histologia com a clínica se fazem necessários para confirmação ou nova formulação de teorias que justifiquem as perguntas às vezes pouco valorizadas ou subestimadas no idoso que interferem na qualidade de vida desta faixa etária.

\section{REFERÊNCIAS BIBLIOGRÁFICAS}

1. Storey A. A functional analyses of sensory units innervating epiglottis and larynx. Expt. Neurol 1968;20:366-83.

2. Sasaki CT \& Weaver EM. Physiology of the larynx. Am J Med 1997;103(5A):9s-18s.

3. Bradley RM. Sensory receptors of the larynx. Am J Med 2000;108(4A):47s-50s.

4. Sanders I, Wu BL, Mu L, Li Y, Biller HF. The innervation of the human larynx. Arch Otolaryngol Head Neck Surg 1993;119:934-39.

5. Wu BL, Sanders I, Mu L, Biller HF. The human communicating nerve: an extension of the external superior laryngeal nerve that innervates the vocal cord. Arch Otolaryngol Head Neck Surg 1994;120:1321-27.

6. Suzuki M. Laryngeal reflexes. In: Hirano M, Kirchner JA, Bless D. Neurolaryngology: recent advances. San Diego: Singular Publishing Group; 1991. p. 142-55.

7. Mortelliti AJ, Malmgreen LT, Gacek RR. Ultrastrutural changes with age in the human superior laryngeal nerve. Arch Otolaryngol Head Neck Surg 1990;116:1062-69.

8. Aviv JE, Martin JH, Jones ME, Wee TA, Diamond B, Keen MS, Blitzer A. Age-related changes in pharyngeal and supraglottic sensation. Ann Otol Rhinol Laryngol 1994;103:749-52.

9. Kirchner JA. Laryngeal proprioception. In: Hirano M, Kirchner JA, Bless D. Neurolaryngology: recent advances. San Diego: Singular Publishing Group; 1991. p. 156-59.

10. Hirano M, Kurita S, Sakaguchi S. Ageing of the vibratory tissue of human vocal folds. Acta Otolaryngol 1989 (Stockh) 107:42833.

11. Sato K \& Hirano M. Age-related changes of elasitc fibers in the superficial layer of the lamina propria of vocal folds. Ann Otol Rhinol Laryngol 1997;106:44-48.

12. Durham CF \& Harrison TS. The surgical anatomy of the superior laryngeal nerve. Surg Gynecol Obstet 1964;118:38-44.

13. Cernea CR, Nishio S, Hojaij FC. Identification of the external branch of the laryngeal nerve (EBSLN) in large goiters. Am J Otolaryngol 1995;16:307-11.

14. Rassekh CH, Driscoll BP, Seikaly H, Laccourreye O, Calhoun KH, Weinstein GS. Preservation of the superior laryngeal nerve in supraglottic and supracricoid partial laryngectomy. Laryngoscope 1998;108:445-47.

15. Dyck PJ. Hypoxic neuropathy: Does hypoxya play a role in diabetic neuropathy? Neurology 1989;39:111-18.

16. Karnovsky MJ. A formaldehyde fixative of high osmolarity for use in electron microscopy. J Cell Biol 1965;27:137.

17. Gundersen HJG. Notes on the estimation of the numerical density of arbitrary profiles: the edge effect. J Microsc 1977;111 pt $2: 219-23$.

18. Sharma AK, Bajada S, Thomas PK. Age changes in the tibial and plantar nerves of the rat. J Anat 1980;130:417-28.

19. Harrison DFN. Fiber size frequency in the recurrent laryngeal nerves of man and giraffe. Acta Otolaryngol 1981;91:383-89.

20. Ogura JH \& Lam RL. Anatomical and phisiological correlations on stimulating the human superior laringeal nerve. Laryngoscope 1953;63: 947-59.

21. Scheur JL. Fibre size frequency distribution in normal laryngeal nerves. J Anat Lond 1964;98: 99-104.

22. Aviv JE. Effects of aging of the pharyngeal and supraglottic areas. Am J Med 1997;103(5A):74s-76s.

23. Mayhew TM \& Sharma AK. Sampling schemes for estimating nerve fiber size: I. Methods for nerve trunks of mixed fascicularity. J Anat 1984;139:45-58.

24. Jacobs JM \& Love S. Qualitative and quantitative morphology of human sural nerve at different ages. Brain 1985;108:897924 . 
25. Rosemberg SI, Malmgreen LT, Woo P. Age-related changes in the internal branch of the rat superior laryngeal nerve. Arch Otolaryngol Head Neck Surg 1989;115:78-86.

26. Dahlqvist A, Carlso B, Hellström S. Fiber components of the laryngeal nerve of the rat: a study by light and electron microscopy. Anat Rec 1982;204:365-70.
27. Burt AM. Neuroanatomia. Rio de Janeiro: Ed. Guanabara-Koogan; 1995.

28. Lima NTMS. Estudo morfométrico das fibras mielínicas do nervo laríngeo recorrente. [Tese Mestrado] USP/Ribeirão Preto; 1995. 